\title{
Sound attenuation devices for dogs barking (estimation of aperture ratio and experimental study of silencer)
}

\author{
Shuichi Sakamoto ${ }^{1}$, Yuichi Toyoshima ${ }^{2}$, Nobuaki Murayama ${ }^{2}$, Toru Miyairi ${ }^{2}$, Akira Hoshino ${ }^{2}$, \\ Takatsune Narumi ${ }^{1}$
}

${ }^{1}$ Faculty of Engineering, Niigata University, Niigata, Japan

${ }^{2}$ Graduate School of Science and Technology, Niigata University, Niigata, Japan

\section{Email address:}

sakamoto@eng.niigata-u.ac.jp (S. Sakamoto)

\section{To cite this article:}

Shuichi Sakamoto, Yuichi Toyoshima, Nobuaki Murayama, Toru Miyairi, Akira Hoshino, Takatsune Narumi. Sound Attenuation Devices for Dogs Barking (Estimation of Aperture Ratio and Experimental Study of Silencer). International Journal of Mechanical Engineering and Applications. Vol. 2, No. 1, 2014, pp. 18-24. doi: 10.11648/j.ijmea.20140201.14

\begin{abstract}
This paper reports a natural ventilation silencer for an animal cage having considerable sound insertion loss. Many veterinary hospitals face a serious problem that hospitalized animals are stressed from the barks of other dogs in the hospital. To address this problem, a solution that barely affects caged dogs' health is required. First, we estimated aperture ratio of the silencer device which was considered by physiological sustainability of dog. Next, we attempted to use a silencer with sound insertion loss by attaching it to an opening side of the cage. We constructed the tested prototype silencers by using the resonance of a rectangular room and a nonwoven fabric. We measured the acoustic characteristics of the silencers that were attached to the real scale model of the animal cage. We also measured their insertion loss by considering the estimated frequency characteristics of the dogs' barking sound. The result shows that proposed silencers have more than $10 \mathrm{~dB}$ insertion loss; therefore, they can maintain respiratory environment of a caged dogs along with good visibility.
\end{abstract}

Keywords: Noise Control, Sound Insertion Loss, Resonance of Rectangular Room, Cell-type Silencer, Nonwoven Fabric

\section{Introduction}

This study is related to the use of natural ventilation silencers for the howling and barking (hereafter referred to as "barking") of dogs. With the spread of nuclear families, low birth rates, and aging populations, pets play an important role in advanced nations. In Japan, the number of complaints and problems caused by the noise created by barking dogs is increasing; it represents the major component of noises in living spaces [1], thus necessitating some sort of countermeasure. In addition, dogs in veterinary hospitals are housed in connecting cages; one dog's barking can cause others to bark as well, creating stress in the other animals in the hospital. One method being considered to remedy this situation is the attachment of a sound insulating board to the opening of the cages and the utilization of forced ventilation. However, the use of sound boards and forced ventilation creates a number of issues, including problems such as hindrance in communicating with animals, noise associated with ventilation intake and output, noise from fans within cages, cost, energy consumption, and the risks of malfunction and power outages; collectively, these problems make this solution unfeasible.

In this paper, we report the evaluation results of sound attenuation of an animal-cage silencer for using dogs' barking model. We focus on cages with an opening on one side, which are commonly used in veterinary hospitals. This method can also be applied to indoor and outdoor animal cages in general households. When asked about the conditions required for a silencer, veterinary hospitals provided the following opinions: "Effective silencing, no burden on the breathing of the animal, two-sided visibility, a thickness that will not inhibit veterinary treatment, and lightweight in order to avoid adding weight to the cage door and burdening workers." To satisfy these conditions, we created a prototype based on resonance within a rectangular chamber divided into cells, adding nonwoven sheets to the interior, tail pipes, and coaxial side branch tube silencers to the open end. We then assessed the sound attenuation performance. 


\section{Experimental Method}

\subsection{Experimental Equipment}

Figure 1 shows the structure of the measurement apparatus. Simulated sound generated by Computer 1 is emitted through a speaker placed within real-size model within the small dog-sized cage. The sound wave was measured using a microphone outside the cage and were recorded and analyzed using Computer 2.

The inner dimensions of the cage were $570 \mathrm{~mm} \mathrm{~W} \times 570$ $\mathrm{mm} \mathrm{H}$ for the opening, with a depth of $700 \mathrm{~mm}$. On the basis of our observations of barking dogs in a veterinary hospital, we placed the speaker in the center of the cage opening at a depth of $200 \mathrm{~mm}$. The microphone was placed above the central axis of the cage at a position $1 \mathrm{~m}$ away from the opening on the outside. Thus, the distance from the front of the speaker to the microphone was $1.2 \mathrm{~m}$. The experiment was conducted in a semi-anechoic room.

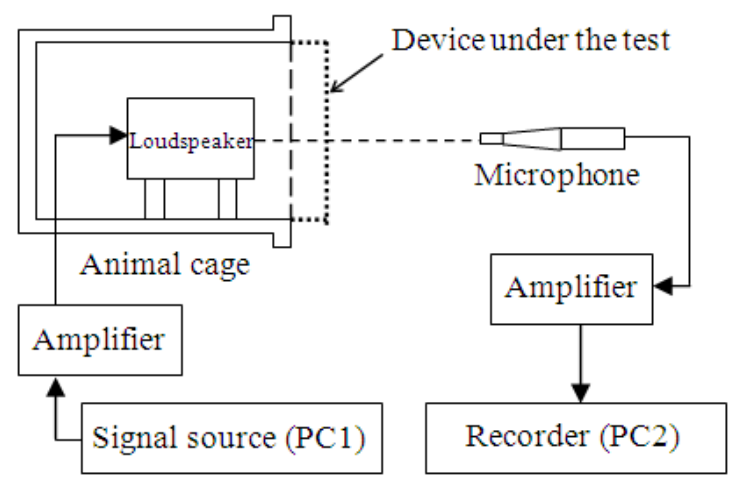

Figure 1. Experimental setup.

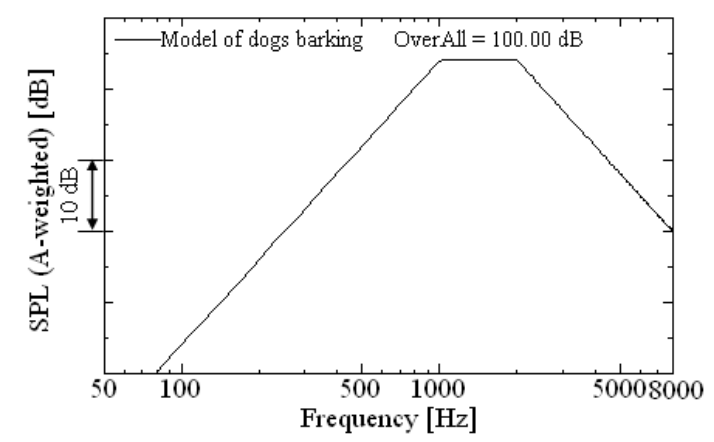

Figure 2. Frequency characteristics model of dog barking (12 dB/oct BPF, logarithmic scale).

\subsection{Frequency Characteristics and Sound Level of Dog Barking}

According to survey results related to dog barking [2], the barks of all breeds have high-level frequency components from 1000-2000 Hz, and sound levels were in the range of approximately $80-90 \mathrm{~dB}$ at a distance of $5 \mathrm{~m}$ from the dog.

In this experiment, we recorded measurements at a position $1.2 \mathrm{~m}$ from the speaker sound source. Because this distance was approximately one-fourth of $5 \mathrm{~m}$, if we consider that the attenuation with distance for a point sound source is $-6 \mathrm{~dB} / \mathrm{dd}$, the sound level is predicted to increase to $12 \mathrm{~dB}$. Thus, we estimated the sound level of the dog at a distance of $1.2 \mathrm{~m}$ to be $100 \mathrm{~dB}$.

Figure 2 shows the dog barking model referencing the frequency characteristics of dog barking. The dog barking model adds frequency weight to flat noise (pink noise) in the graph where the frequency axis is logarithmic. For the added frequency weight, the attenuation characteristic was assumed to be approximately $12 \mathrm{~dB} /$ oct for frequencies less than $1 \mathrm{kHz}$ and greater than $2 \mathrm{kHz}$. The sound level (overall value) was set to $100 \mathrm{~dB}$.

Figure 3 shows the dog barking model, shown in logarithmic scale on the frequency axis in Fig. 2, converted to a linear scale. This result shows that the $3 \mathrm{~dB} /$ oct decreasing frequency characteristic of pink noise is included. Figure 3 includes frequencies up to $8 \mathrm{kHz}$; however, in linear frequency analysis with $24 \mathrm{kHz} / 512$ line resolution, as was the case with the measurement apparatus used, we set the level values for each frequency such that the overall value of the dog barking model was $100 \mathrm{~dB}$.

Because the bark of a dog is difficult to reproduce, we used simulated sound output from the speaker. Figure 3 shows the results for simulated sound output from the speaker and measured with the microphone. These results show that the speaker frequency characteristics are in superposition with the simulated sound measured. Using the difference in the frequency characteristics of the simulated sound and the dog barking model shown in Fig. 3, we equalized the measurement results acquired after the silencer was attached. This approach allowed us to obtain measurement results equivalent to the dog barking model, simulated sound. We hereafter refer to this sound attenuation of the dog barking model simply as "sound attenuation."

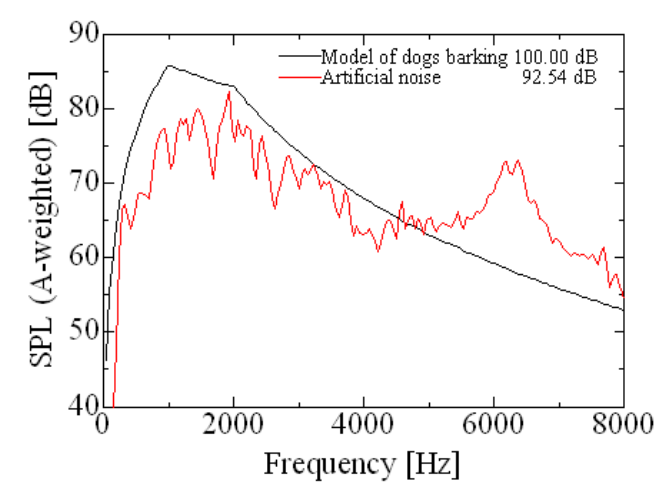

Figure 3. Model of dog barking (12 dB/oct BPF, linear scale) and artificial noise.

\subsection{Determination of the Aperture Ratio of the Silencer}

Natural ventilation in buildings occurs depending on wind and the indoor/outdoor temperature difference [3]. Ventilation due to buoyancy occurs between the still indoor air and the animal cages on the basis of temperature difference. The relationships between the required 
ventilation flow rate for the dog cages [4] and the temperature difference needed for natural ventilation are provided in (1):

$$
Q_{R}=Q=\alpha A \sqrt{2 g\left(h_{2}-h_{1}\right)\left(\frac{t_{i}-t_{0}}{273.15+t_{i}}\right)}
$$

Where the required ventilation flow rate $\mathrm{QR}$ is determined considering the dog's physiological sustainability [5] [6] using (2) [7]:

$$
Q_{R}=\frac{60}{T} V
$$

The permitted $\mathrm{CO} 2$ concentrations according to the "Act on Maintenance of Sanitation in Buildings" and the "Japan Society for Occupational Health Standards" are 1000 and 5000 ppm, respectively. Using these respective values, we calculated the required ventilation flow rate.

The flow rate coefficient $\alpha$ is an opening characteristic that combines the total pressure loss of entrance and exit (i.e., the pressure loss due to profile drag and frictional pressure loss) and is given by the following formulae [3].

$$
\begin{gathered}
\alpha=\frac{1}{\sqrt{\xi_{1}+\lambda \frac{l}{D}+\xi_{2}}} \\
D=2 \sqrt{\frac{A \times r}{\pi}}
\end{gathered}
$$

The shape of the opening on the front face of the silencer is shown in Fig. 4. The effective aperture area for multiple openings arranged in parallel is given in (5):

$$
(\alpha A)_{12}=\alpha_{1} A_{1}+\alpha_{2} A_{2}
$$

Because the produced silencer consists of 16 cylindrical openings in an array formation, as shown in Fig. 4, the effective aperture area is 16 times the effective aperture area for any single opening. The profile drag coefficients are given as $\xi_{1}=0.5$ and $\xi_{2}=1.0$ [8].

Here, if we solve for $t_{\mathrm{i}}-t_{\mathrm{o}}$ in (1), we obtain the relationship between the aperture ratio and the required temperature difference, as given in (6):

$$
t_{i}-t_{0}=\frac{\left(\frac{2 Q_{R}}{\alpha A \times r}\right)^{2}\left(273.15+t_{i}\right)}{2 g\left(h_{2}-h_{1}\right)}
$$

The average head of the breathing cycle $h_{2}-h_{1}$ is given as half the cage height, $285 \mathrm{~mm}$. Cylinder length 1 is given as $150 \mathrm{~mm}$, which is the maximum length of the silencer used in this study.

Figure 5 shows the observed relationship between the aperture ratio and the required temperature difference. To sufficiently minimize the required temperature difference and maintain visibility between the animals and humans, we used an aperture ratio of $25 \%$. Figure 4 is illustrated with an aperture ratio of $25 \%$.

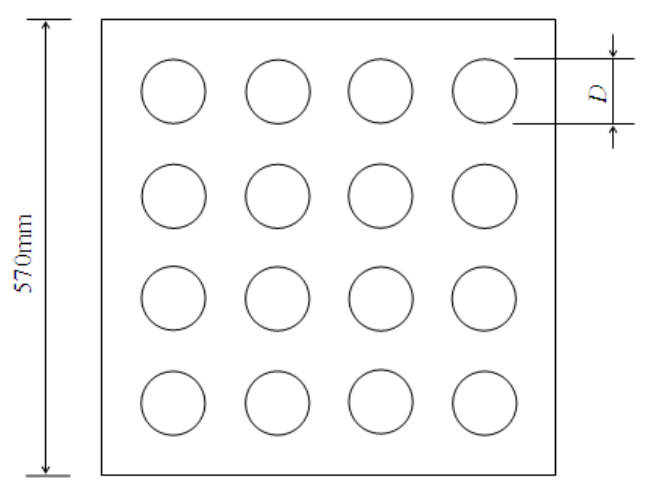

Figure 4. Apertures on the front face of cell-type silencer (aperture ratio: $25 \%, D=80 \mathrm{~mm}$ ).

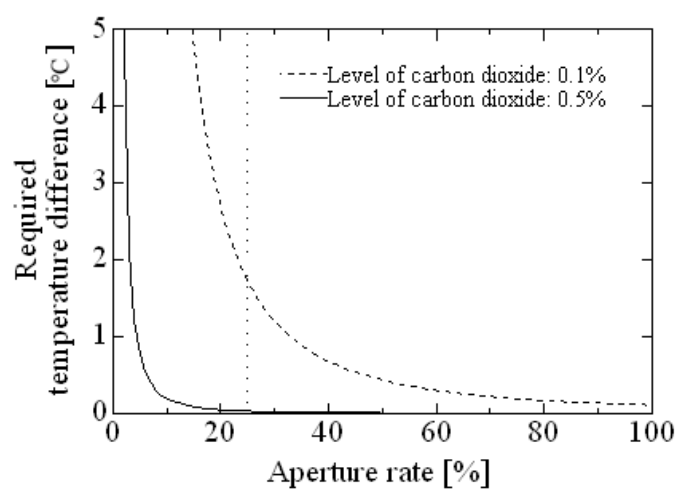

Figure 5. Relationship between required temperature difference and aperture ratio.

\section{Silencer Prototype and Experiment}

\subsection{Basic type of the Silencer (Attenuation in Rectangular Chambers with Cylindrical Openings)}

Figures 6 and 7 show a silencer with rectangular chambers arranged in an array configuration with openings on both sides. The diameter of the opening is set to a size through which a human hand can pass to allow communication with the animal through touch and provide food.

When the aperture ratio is set to $25 \%$ with a $4 \times 4$ arrangement of 16 silencers and a material thickness of 4 $\mathrm{mm}$, an interior side of the square section of the cell $\left(l_{y}=l_{z}\right)$ is $137.5 \mathrm{~mm}$ and the diameter of the opening is $80 \mathrm{~mm}$. This diameter is sufficiently large to accommodate all, but the largest Japanese hands. Corrugated plastic (material: polypropylene, thickness: $4.0 \mathrm{~mm}$, area density: 0.54 $\mathrm{kg} / \mathrm{m}^{2}$ ) was used for material of these prototype silencers.

Figure 8 shows the results of experiments conducted with this basic-type silencer. A sound attenuation of approximately $4 \mathrm{~dB}$ was obtained. Because the inner dimension of the length of the diagonal line observed from the front of the cell is $194 \mathrm{~mm}$, a plane wave in the thickness direction of the silencer is thought to be produced 
up to approximately $1 \mathrm{kHz}$. Thus, for frequencies up to approximately $1 \mathrm{kHz}$, we can obtain an expansion silencing effect by suddenly shrinking or expanding the cross-sectional area in front of and behind the opening. The attenuating peak frequency of expansion silencer is known as the function of length of silencer $(1 \mathrm{x}=92 \mathrm{~mm})$. In this case, the first attenuating peak is calculated approximately $920 \mathrm{~Hz}$, and we can also see attenuation around $900 \mathrm{~Hz}$ in Fig. 8 .

In addition, multiple rectangular chamber resonance modes probably occur at frequencies greater than $1 \mathrm{kHz}$. Resonance frequencies of rectangular chamber are calculated by well-known (7). Each fundamental frequencies of direction of $l_{y}$ and $l_{z}$ mode and diagonal line mode of $l_{y}$ and $l_{z}$ are calculated $1250 \mathrm{~Hz}$ and $1770 \mathrm{~Hz}$, respectively. In Figure 8, we can also see a sound attenuation effect due to energy dispersion caused by these resonances.

$$
f=\frac{c}{2} \sqrt{\left(\frac{n_{x}}{l_{x}}\right)^{2}+\left(\frac{n_{y}}{l_{y}}\right)^{2}+\left(\frac{n_{z}}{l_{z}}\right)^{2}}
$$

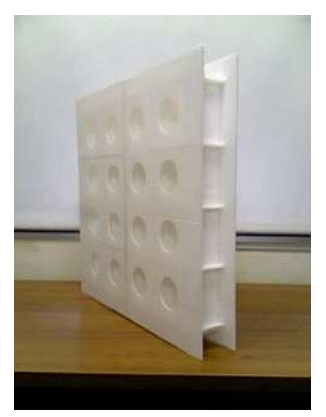

Figure 6. Photograph of the basic-type silencer, $l_{x}=92 \mathrm{~mm}$.

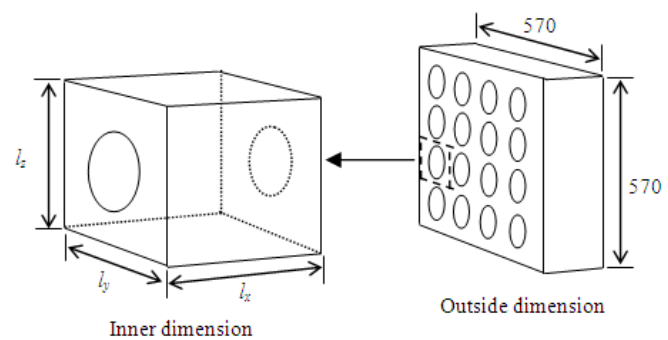

Figure 7. Schema of the basic-type silencer, $l_{x}=92 \mathrm{~mm}, l_{y}=l_{z}=137.5 \mathrm{~mm}$.

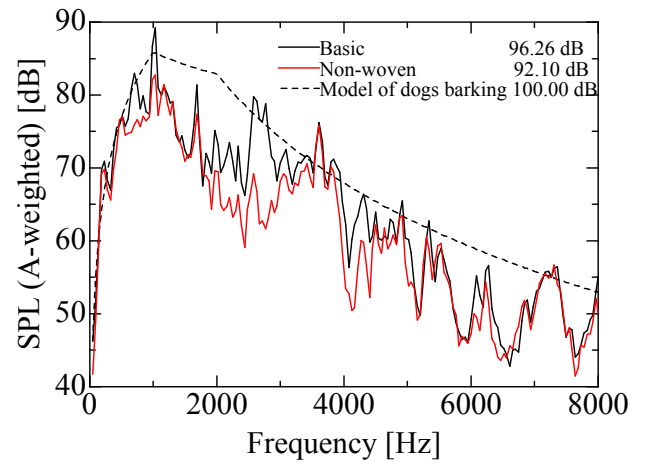

Figure 8. Equalized attenuation of basic type (with or without nonwoven fabric).

\subsection{Attachment of Sheets of Nonwoven Fabric}

We conducted experiments with a silencer that linked the openings of the basic silencer discussed in the previous section with cylindrical nonwoven fabric sheets as shown in Fig. 9. This measurement result was shown in Fig. 8. Through the back air space effect of the nonwoven fabric [9] as shown in Fig. 10, sound attenuation increased in comparison with that achieved with the basic silencer in a wide frequency range of 1000-3000 Hz. We achieved sound attenuation of approximately $8 \mathrm{~dB}$. Compared with the attenuation achieved with the basic silencer, the attachment of the nonwoven fabric sheets increased sound attenuation by approximately $4 \mathrm{~dB}$, making this an effective countermeasure.

This phenomenon can be explained as follows. As well known, the sound particle velocity near a rigid wall has the maximum value at a distance of $1 / 4$ wavelength from there; therefore, the sound absorption coefficient must be greatest when the top surface of the body of a sound-absorbing material coincides with this position [10].

To confirm as mentioned above, we measured the normal incident sound-absorption coefficient of nonwoven fabric sheets with back air space based on ISO 10534-2. The composition of the apparatus is shown in Fig. 11. In the measurement, a Brüel \& Kjær type 4206 2-microphone impedance measurement tube was used. Thicknesses of back air spaces form nonwoven fabric sheets were set to 57 $\mathrm{mm}$ and $29 \mathrm{~mm}$ which are shown in Fig. 10.

Figure 12 shows results of measurements. In both cases, high absorption coefficient over the target frequency range was obtained.

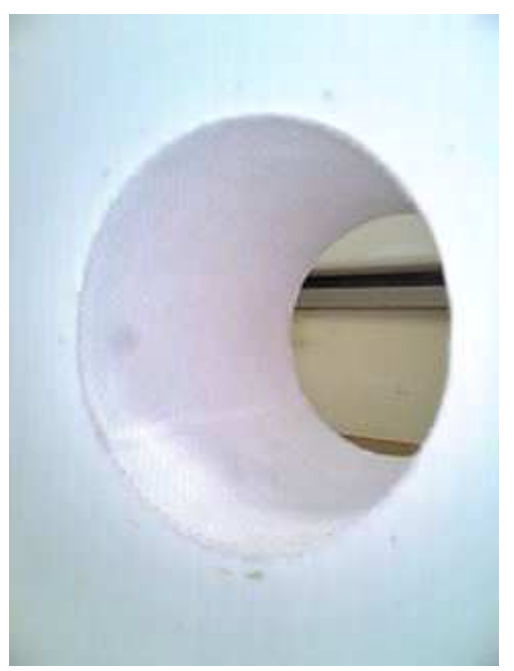

Figure 9. Partial photograph of nonwoven fabric attached between both side openings (specifications of nonwoven fabric sheet; code: $3 \mathrm{A01A}$, material: polyester, thickness: $0.39 \mathrm{~mm}$, area density: $100 \mathrm{~g} / \mathrm{m}^{2}$, manufacturer: Toyobo. co.).

\subsection{Addition of a Tail Pipe to the Opening}

In this section, we present the results of silencing by throttle effect using tail-pipe. 


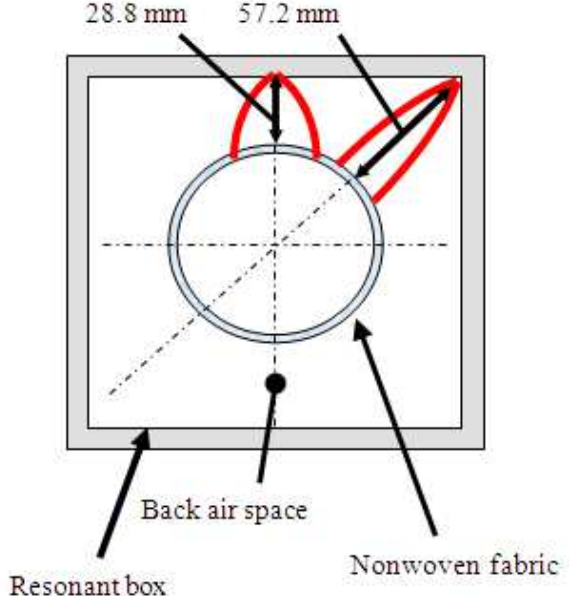

Figure 10. Shortest and longest back air space on the section of $y$-z plane.

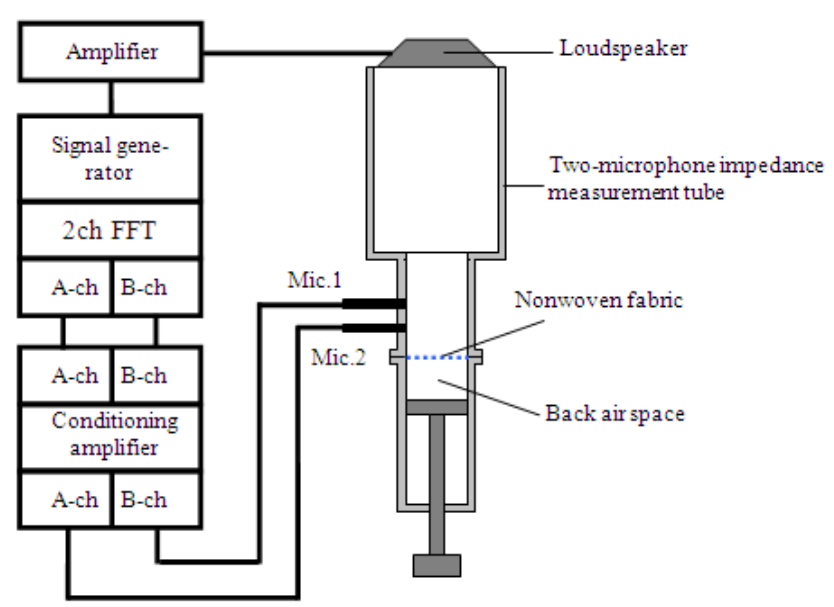

Figure 11. Two-microphone impedance measurement tube system.

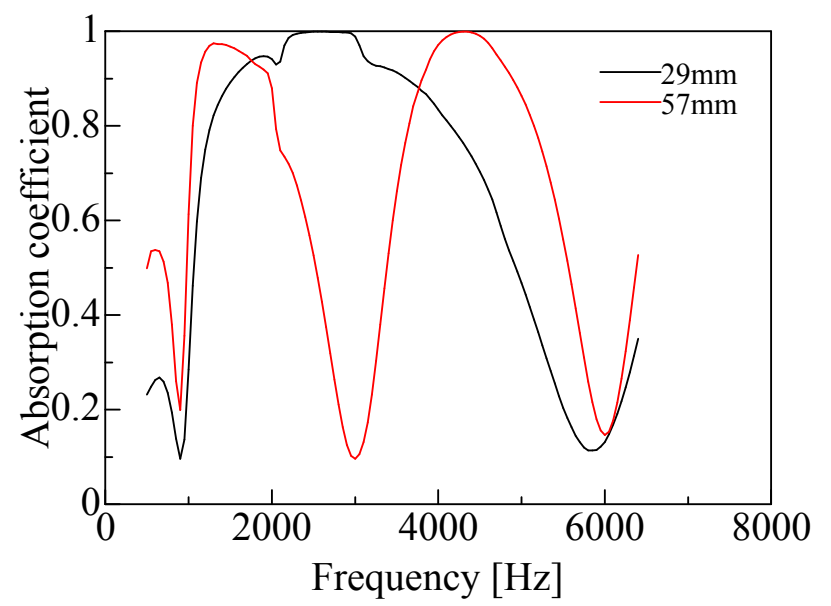

Figure 12. Normal absorption coefficient of nonwoven fabric with back air space (back air space: $29 \mathrm{~mm}, 57 \mathrm{~mm}$ ).

Figure 13 shows a photograph of a tail pipe added to the opening of the silencer. The tail pipe was constructed from a poly(vinyl chloride) pipe with an internal diameter of 83 $\mathrm{mm}$, a thickness of $3.0 \mathrm{~mm}$, and a length of $50 \mathrm{~mm}$. The tail pipe with an internal diameter of $83 \mathrm{~mm}$ formed a plane wave at frequencies up to approximately $2 \mathrm{kHz}$, and we treat this as an acoustic tube in this frequency range. We calculated the attenuating peak frequency of the throttle effect by the tail pipe as both ends opened using (8). Here, we calculated the open-end correction length using $E=0.4$ for orifice [11] through (9).

$$
\begin{gathered}
f=\frac{m c}{4\left(l_{t}+\Delta l_{1}+\Delta l_{2}\right)} \quad \mathrm{m}=1,3,5, \cdots \\
\Delta=a E
\end{gathered}
$$

According to (9), open-end correction length for both ends is $16 \mathrm{~mm}$. Therefore, the length of an effective tail pipe $\left(l_{t}+\Delta l_{1}+\Delta l_{2}\right.$ in (8)) can be estimated from this total to be $82 \mathrm{~mm}$. According to (8), the first attenuating peak frequency of the tail pipe is estimated to be approximately $1050 \mathrm{~Hz}$. In addition, because a plane wave does not form within a tail pipe at the third attenuating peak frequency, which is three times of the fundamental frequency of 1050 $\mathrm{Hz}$, the third attenuating peak does not occur.

Measurement results are shown in Fig.s 14 (basic configuration + tail pipe) and 15 (basic configuration + tail pipe + nonwoven fabric sheet). In all cases, sound attenuation was large at around $1000 \mathrm{~Hz}$; and these results are well matched to the previously predicted attenuating peak frequency. Also, the addition of the tail pipe was observed to increase sound attenuation by approximately 2 $\mathrm{dB}$, irrespective of the presence of the nonwoven fabric.

\subsection{Addition of Side Branch Tube Silencers on the Same Axis to the Opening}

Here, we discuss a silencer in which grating the same length as the tail pipe discussed in the previous section is added. As presented in Fig. 16, the donut-shaped cross-section pipe fitted along the same axis between the tail pipe and grating is open on one end and closed on the opposite end. This arrangement serves as a side branch silencer installed on the same axis as the tail-pipe opening.

Figure 17 shows the tail pipe and grating expressed as an electrical equivalent circuit. The open end of the tail pipe expressed with a four-terminal network transfer matrix is terminated with radiation impedance at the open end and incident impedance of the side branch tube. At the resonance frequency of the length of the side branch tube (here, the depth of the grating), the impedance is smaller at the open end, and we expect a sound absorption effect.

Figure 18 shows measurement results. Sound attenuation was improved in the range $1-2 \mathrm{kHz}$ centered on $1200-1400$ $\mathrm{Hz}$. Sound attenuation for the dog barking model was achieved because the $1700-\mathrm{Hz}$ peak was reduced, and we obtained $1.2 \mathrm{~dB}$ sound attenuation in comparison with that achieved in the previous section through the addition of the grating. We achieved sound attenuation of $11.01 \mathrm{~dB}$ for the dog barking model, which exceeds our initial target of 10 dB. 


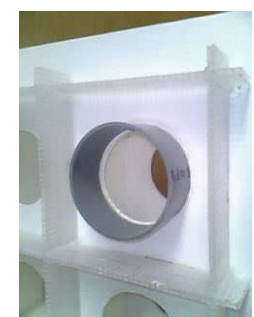

Figure 16. Partial photograph of tail tube surrounded by grating.

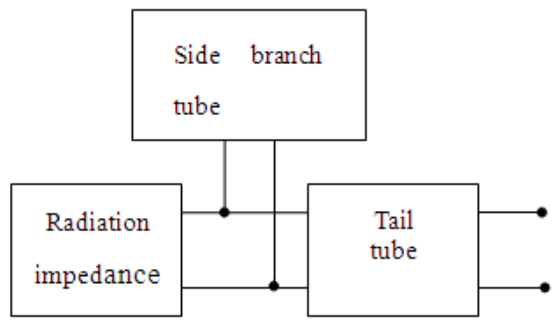

Figure 17. Equivalent circuit for open end of tail tube (terminated in two impedances).

To confirm as mentioned above, we performed experiments and calculations of the normal incident transmission loss for the co-axial arranged side-blanch silencer. In the experiments, a Brüel \& Kjær type 4206T 4-microphone impedance measurement tube was used to measure the normal incident transmission loss based on ASTM E2611-09. Figure 19 shows cross-sectional schema of the test sample construction with concentric side-blanch tube. A tail pipe (inner diameter $14 \mathrm{~mm}$, length $60 \mathrm{~mm}$ ) was attached to a throttle which inserted in an impedance measurement tube of inner diameter $29 \mathrm{~mm}$. Calculated value was estimated using single dimensional transfer matrix method [12]. In this test sample, the length of tail pipe was $60 \mathrm{~mm}$ which is $10 \mathrm{~mm}$ longer than that of in Fig. 16 , because of estimating end correction value for donuts shaped open end as shown in Fig. 16 is difficult.

These results are shown in Fig. 20, and both trends are fairly matched each together. Thus, it was shown that the co-axial side blanch will operate just as general side-blanch silencer. Moreover, frequency range of attenuation in Fig. 20 was appeared also in Fig. 18.

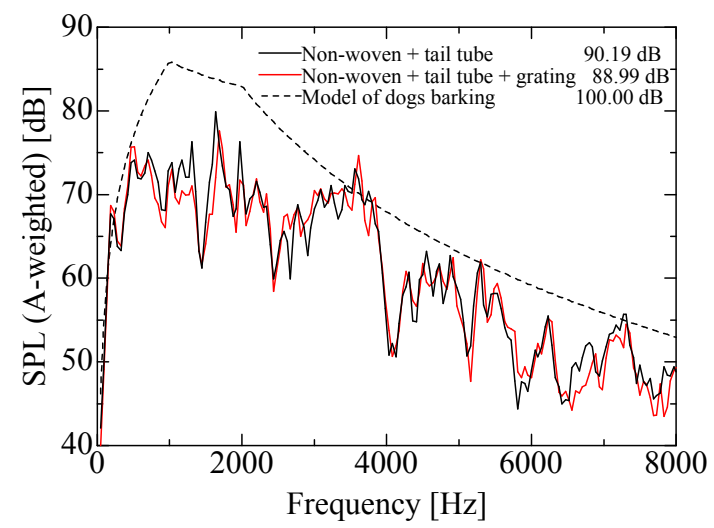

Figure 18. Equalized attenuation with nonwoven fabric and tail tube (with or without grating).

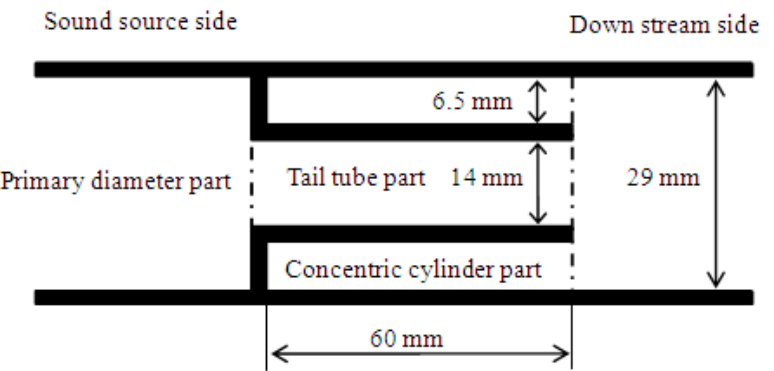

Figure 19. Cross section of test sample for transmission loss measurement (tail tube surrounded by concentric cylinder).

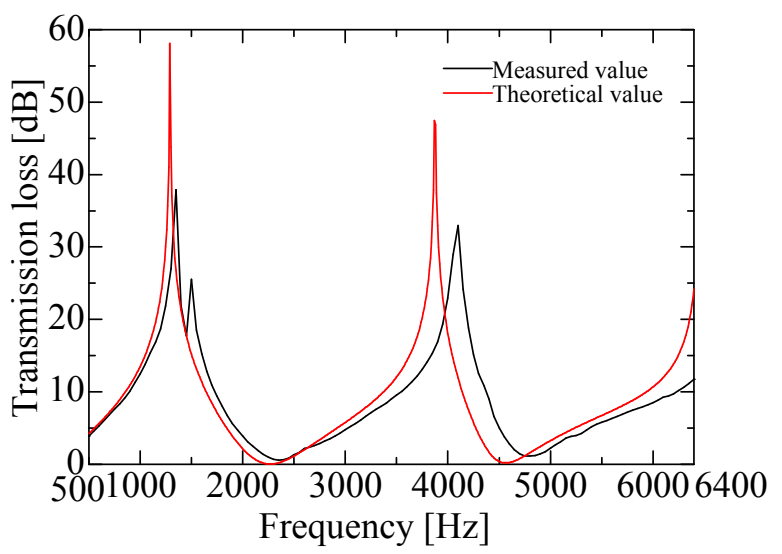

Figure 20. Comparison of transmission loss between calculation [12] and measured value of test sample in fig. 19.

\subsection{Summarizing Sound Attenuation for the Dog Barking Model}

The results obtained for sound attenuation for the dog barking model are summarized in Table 1 . The addition of a tail pipe improved sound attenuation by approximately 2 $\mathrm{dB}$, irrespective of the presence of nonwoven fabric sheets. Also, the addition of nonwoven fabric sheets to either the basic silencer or the silencer with a tail pipe attached resulted in an improvement in sound attenuation by approximately $4 \mathrm{~dB}$.

Table 1. Equalized attenuation under various conditions [dB].

\begin{tabular}{lll}
\hline & $\begin{array}{l}\text { Without } \\
\text { nonwoven fabric }\end{array}$ & With nonwoven fabric \\
\hline $\begin{array}{l}\text { Rectangular room only } \\
\text { (basic type) }\end{array}$ & 3.74 & 7.90 \\
$\begin{array}{l}\text { Basic with tail tube } \\
\begin{array}{l}\text { Basic with tail tube and } \\
\text { grating }\end{array}\end{array}$ & 5.67 & 9.81 \\
\hline
\end{tabular}

\section{Conclusions}

We conducted experiments with silencers for application to cage openings to alleviate the noise of dogs barking, and we obtained the following results:

We estimated aperture ratio of the silencer device which was considered by physiological sustainability of dog.

Silencers based on rectangular chamber resonance achieved sound attenuation in a sufficiently wide frequency 
range through resonance in a variety of modes.

Silencers based on rectangular chamber resonance resulted in greater sound attenuation effects across a wide frequency range through the addition of nonwoven fabric sheets.

The addition of a tail pipe to the opening of the rectangular chamber resulted in sound attenuation effects because of the throttle effect.

The addition of grating to the opening corresponded with the parallel connection of concentric side branch tubes for open end radiation impedance, which resulted sound attenuation effects through the resonance of a tube with one closed side corresponding to the depth of the grating.

By adopting a combination of the previously described silencer structure, we fulfilled the requirements for an aperture ratio that is physiologically sustainable for dogs, allows good visibility into the cage, allows hand access, and is light in weight, with a thickness of $150 \mathrm{~mm}$. We simultaneously achieved sound attenuation results of greater than $10 \mathrm{~dB}$ for the dog barking model.

\section{Nomenclature}

$A$ : Total aperture area of cage $\left[\mathrm{m}^{2}\right]$

$a$ : Inner diameter $0.04 \mathrm{~m}$

$c$ : Velocity of sound in air $[\mathrm{m} / \mathrm{s}]$

$D$ : Diameter of aperture [m]

$E$ : Open end correction

$f$ : Frequency $[\mathrm{Hz}]$

$g$ : Acceleration of gravity $\left[\mathrm{m} / \mathrm{s}^{2}\right]$

$h_{1}$ : Height of air exit [m]

$h_{2}$ : Height of air entrance [m]

$l$ : Length of circular tube [m]

$l_{x}, l_{y}, l_{z}:$ Dimension of rectangular chamber for each direction $[\mathrm{m}]$

$l_{t}$ : Length of tail tube [m]

$n_{x}, n_{y}, n_{z}$ : Order of mode for each direction

$Q$ : Ventilation flow rate $\left[\mathrm{m}^{3} / \mathrm{s}\right]$

$Q_{R}$ : Required ventilation flow rate $\left[\mathrm{m}^{3} / \mathrm{s}\right]$

$r$ : Aperture ratio

$T$ : Time at maximum respiration until the cage reaches permitted $\mathrm{CO}_{2}$ concentration [s]

$t_{i}$ : Internal temperature of cage $\left[{ }^{\circ} \mathrm{C}\right]$

$t_{0}$ : External temperature of cage $\left[{ }^{\circ} \mathrm{C}\right]$

$V$ : Internal volume of cage $\left[\mathrm{m}^{3}\right]$

$\alpha:$ Flow rate coefficient

$\alpha A$ : Effective aperture area $\left[\mathrm{m}^{2}\right]$

$\Delta l_{1}, \Delta l_{2}$ : Open end correction length for each end [m]

$\xi_{1}$ : Coefficient of entrance loss

$\xi_{2}$ : Coefficient of exit loss

$\lambda$ : Coefficient of frictional pressure loss

\section{References}

[1] N. Hashimoto, Questionnaire on neighborhood noise dispute to person in charge of noise problem in municipal office, AIJ Journal of Technology and Design, Vol. 13, No. 25, 2007, pp 171-176.

[2] Ministry of environment (Japan), The 3rd investigative committee of the state of protection management of animal (Document 2 Occurrence situations of harms and troublesome problems), Government of Japan, 2004, pp. 19-21.

http://www.env.go.jp/nature/dobutsu/aigo/2_data/arikata/h16 _03/mat03.pdf

[3] "Air-conditioning / Sanitary Engineering Handbook" Vol.1, Edited and published by Society of Heating, Air-Conditioning and Sanitary Engineers of Japan, 1963, pp. 320-321, NCID: BA53189532.

[4] Society of researches for textbook of environment engineering, "Textbook of Environment Engineering", Shokokusha publishing co., ltd., 2000, pp. 134-143, ISBN: 4395005160 .

[5] M. Tsuboi, "Dog Anatomy and Table for Physiological Calculation", Bunkyo-shoin, 1971, p.195, NCID: BN06961627.

[6] T. Tsuda,."Domestic Animal Physiology", Yokendo, 2004, p. 133, NCID: BA66764054.

[7] M. Miura, “Architectural Environment Engineering", Morikita Publishing Co., Ltd., 2006, pp. 285-286, ISBN: 9784627581111.

[8] U. Inoue, "Duct Calculation Handbook", Kurimoto, Ltd., 1962, pp. 121-122, NCID: BN11470089.

[9] S. Sakamoto, T. Miyairi, A. Hoshino, I. Kourakata, T. Sato, "Attenuation characteristics of double orifice silencer jointed by permeable membrane (measurement and theoretical analysis of sound transmission loss)", Transactions of the Japan society of Mechanical Engineers, Series C, Vol. 79, No. 806, 2013, pp. 3474-3486.

[10] Z. Maekawa, M. Morimoto, K. Sakagami, "Architectual • Environmental Acoustics 2nd edition", Kyoritsu publishing CO., LTD., 2000, pp. 80-81.

[11] R. H. Bolt, S. Labate, U. Ingård, "The acoustic reactance of small circular orifices", J. Acoust. Soc. Am. Vol. 21, No. 2 , 1949, pp. 94-97.

[12] H. Sasao, "A guide to acoustic analysis by Excel - Analysis of an acoustic structural characteristic - (4) Analysis of the duct system silencer by Excel", Journal of the Society of Heating, Air-Conditioning and Sanitary Engineers of Japan, Vol. 81, No. 1, 2007, pp. 51-58. 